\title{
Trends of building and accessing digital collections and problems of digital divide in the emerging digital era
}

\author{
Dr. I.V. Malhan \\ Professor and Head \\ Department of Library Science, \\ University of Jammu, \\ Jammu (Tawi)-180006, India \\ e:mail - imalhan 47@rediffmail.com
}

\begin{abstract}
The instant accessibility of information through the Internet and development of the World Wide Web have accelerated digitization work in libraries publishing houses and in enormous other organizations concerned with collecting, storing and communication of information. The paper depicts reasons for growing acceptability of digital collections and new kinds of challenges the digital media poses for the libraries and information centres. Portrays the growing unbundling of information in digital form and indicates the trends of development of new information products and knowledge management tools in the emerging digital environment. The growing digitization of information has created an information explosion to a level which the humanity has never witnessed before. In such a scenario cooperation, collaboration, synergy and convergence of work and efforts are the means to ensure accessibility of best possible information services to user communities. The growing volume of digitized information have bridged the information accessibility gap for some by providing access to enormous volume of information on desktop but it has created the problem of digital divide for those who have no access to information technology infrastructure or lack competencies to effectively use it. It suggests that a massive collaborative effort is required for imparting information literacy to people to build their capacities to derive adequate benefits from the digital revolution.
\end{abstract}

\section{Introduction}

The growth of the Internet and development of the web have offered new means for instant access to information and accelerated the process of digitization of information. Because of elimination of need for physical storage of information, better appeal and comprehension of multimedia digital documents, to their economical transmission at fast speed and possibility of using the same document by several users at the same time, the network transmitted digital information collections are increasingly accepted as the medium for recording and transmission of information. Digital collections have helped to realize the goal of universal availability of publications in the true sense. Besides overcoming the barriers of time and space, digital documents have overcome the language barrier as recorded information can be converted from one language to another using he Unicode Metadata Methodology. It has also helped to overcome the 
terminological barrier as software tools are available to convert the traditional terminology to the equivalent internationally standardized terminology.

The new digital information environment offers immense opportunities for sharing and leveraging from the knowledge of each other. By integration of disparaged knowledge resources spread all over the world, it has helped to speed up the process of research and thus helped in the further growth and modification of knowledge. It has led to diffusion of cultures and by linking information with information helped to develop insights and realize people their true potential. Digital revolution in fact has converted the world into a global village, increased opportunities and competition and influenced almost every component of society. Just in time decision making, teleconferencing, telemedicine, e-commerce, e-learning are just some areas of wide range of developments. The life cycles of industrial products have drastically shortened and smart ways of marketing emerged.

The digitized information revolution is made possible by the fast track developments in information technology and is fueled by the Internet. Digitization of collections is giving new life to deteriorating manuscripts and faded paintings and pictures. The hidden treasures of knowledge which remained inaccessible for centuries are now made available to humanity after their digitization. Digitization of print collections and new digital documents made beginning of a movement for free open access availability of information resources. This process on the whole has facilitated delivery of more information to greater number of information users and provided more choices for decision making and problem solving. The advancing digital technologies facilitated codification of traditional knowledge that remained scattered in cocooned communities, isolated groups, individuals, rare manuscripts and personal diaries.

Digital collections accessible in the networked environment have democratized the process of information access. Everyone having connectivity to the Internet can access the same information at the same time using search engines. Scientists in the developing countries can access research journals at the same time their counterparts do in the developed countries. Individuals now enjoy greater freedom of expression as they can express their views through blogs that are also partially helping to convert tacit knowledge into explicit knowledge. "The Digital -audio-based Information System (DAISY) Consortium facilitates the use of information technology by the world's blind and print disabled population. Developing countries can become members of the DAISY Consortium. The project NOVA (Non-visual access to the digital library), undertaken by the Centre for Research in Library and Information Management, Manchester Metropolitan Universities, is concerned with digital library services to blind and visually impaired people". (Malhan and Gulati, 2003, p.211) Efforts are on to organize outreach programs to help disadvantage people and rural communities to access information sources (eg.e-choupals in India).

Digitized collections of information however have further accelerated the growth of information. A study conducted at the University of California, at Berkeley has shown that computers and the Internet has created a tremendous information explosion. "It has taken 300,000 years for humans to accumulate 12 exabytes (1 billion gigabytes) of information. It will take 2-5 more years for humans to accumulate the next 12 exabytes (Computer Today, 2000, p.27). The large scale digitization of information also led to unbundling of information. The ocean of unbundled information has posed the challenge of information tracking and information mining. Getting more hits does mean one has 
access to better quality information sources too. Selection, evaluation, quality filtering, warehousing and trafficking of information is another kind of massive work involved. "To-day our capacity to record information has increased exponentially overtime while the longevity of the media to store information has decreased equivalently (Conway). Scientific validation of information collected, veracity of facts, authentication of the traditional digitized knowledge and its integration with the existing scientific knowledge, obsolescence of information as well the storage technology are the new challenges. The storage and use of digital collections are dependent on machines and software tools which they themselves change with the passage of time. In order to continue the preservation of important information resources, migration of data from one set of technologies to another, hypermedia organization and development of user friendly guides to the Internet hosted digital information resources are other problems.

Just like machines, some information sources also become outdated with new value addition and updating. "As digital materials become dated, dead links replacement will be determined by the appropriate staff members, who will determine whether or not:

- The item is still available and can be replaced;

- Another item or format might better serve the same purpose;

- There remains sufficient need to replace the same item:

- Updated, newer or revised materials better replace a given item:

- The item has historical value;

- Another network agency could better provide that or a comparable item" (4).

In the fluid information environment building digital collections is not an easy task. Some authors are writing and reporting their works chapter by chapter and even page by page and hosting their views hour by hour (sometimes less than this time). Some bookstores started selling books chapter by chapter and even page by page (amazon.com's announcement). However, there is great opportunity to organize better current awareness services.

\section{New products and new generation of information tools.}

The large scale digitization and unbundling of information has given birth to a variety of aggregators and information access tools e.g. J-Gates, Ingenta.Com, Moreover (Aggregator of blogs), Scopus, Science Direct. It has also offered immense potential to generate a variety of information products in commercial and non commercial sectors e.g. PubMed, MD Consult, SKOLAR, MD, OCLC's First Search, MD Consult's First Consult, $24 \times 7$ Learning, etc. A variety of new generation information and knowledge management tools are also emerging which help in content creation, content extraction, management and retrieval. e.g. Ulrich Serials Analysis System is a suitable tool to analyze and rationalize serial. Collections; Ficstar (5) webgrabber helps to extract data from Google, Yahoo, amazon.com, monstor.com, e-commerce sites and all kinds of member lists. Jamine Milne (2004) reported Headline grabbing devices which help to track and control global news sources. Web Navigation Description Language (WNDL) ias an XML based tool aimed at intellectual property researchers. A number of blogging tools such as Blogger, Live Journal, Moveable Type, Type pad, slashcode, Wordpress, GreyMatter are available. There are numerous other information products and knowledge management tools developed and some are being continuously improved 
with value addition and more user friendly features. Some of the agencies are even collaborating and converging their efforts for bringing out more comprehensive and more effective information tools e.g. PubMed and Biomed central collaboration, Wilsondata bases link to Scirus.

\section{Digital collections and emerging professional issues.}

A number of digital libraries have been successfully created and enormously used e.g. Library of Congres's ten million digitized collections that are used by billions of users every year, Carnegie Mellon University and Indian Institute of Science, Bangalore's ongoing million books project. Traditional knowledge Digital Library (TKDL) developed by NISCAIR and the Department of Ayurveda, Yoga and Naturopathy, Unani, Sidha and Homeopathy, is and excellent example of the codified traditional knowledge on Indian System of Medicine. A good initiative in the collection of theses and dissertations is Networked Digital Library of Theses and Dissertations where Vidhyanidhi from India is participating. There are many other examples such as International Children Digital Library, Alexandria Digital Library, the British Library Digital Library, the Digital Library of MIT theses. It is not possible to list all here. Digital libraries of special kinds of material are also emerging e.g. Digital Gallery hosts 2,75,000 pictures digitized by he New York Public Library and Ingenious aims to bring together 30,000 images and viewpoints taken from the British museums. We are operating in a flood of digital information environment:

- Where many challenges abound us but opportunities also surround us.

- Where we are facing instability but not insecurity.

- Where we are facing competition from other information providers.

- Where we require constantly watching the technological developments and continuously training ourselves in appropriate technology handling.

- Where we require developing user focused information services and constantly add value to these services.

- Where we require synergizing our existing efforts and proactively and collaboratively work to better serve our users.

- Where we must add 'humanism' and not 'commerce' to our work efforts and services.

With growing digitization of documents the issues before the profession are:

- Increasing scarcity of time with user communities.

- Declining reading habits and shifts from reading for pleasure to reading for a purpose.

- Busy user communities' shift from reading elaborate reports to consulting.

- Identification of what worthwhile information is available in the open access sources.

- Legal deposit of digital documents (Project Paradigm) and creation of mirror sites.

- Frame policies for what to digitize and retain.

- Collaboratively search, evaluate and quality filter the newly born information. 
- Shift from procedure based to user based library policies.

- Develop state of the art schemes and knowledge management tools for organization of digitized information.

- Work on cost effective ways to acquire and access useful and need based digital collections through library consortia.

- Develop better user interfaces as technology advances.

- Work on plans to constantly fill the skill gap of staff engaged in libraries and information centres.

- As far as possible facilitate free access to ICT infrastructure and encouraged free services to users.

- Explore the possibility of collaborative arrangements with other agencies in the best interest of digitized knowledge management and dissemination.

- Empower end users by organizing all possible need based information literacy training programs for users.

Library and information professionals must recognize the rapid changes in the society at large and changing information patterns of their user communities. People prefer fax or email over postal letters and mobile telephony is getting priority over fixed phones. No one has time to sit near the story telling folklore as every one wants to search the Internet explorer. In the growing hectic life style people generally search and use information pertaining to what affects them most? What interests them most? What improves their performance? What meets their objectives and purposes? Libraries therefore must remodel their operations, re-engineer their services and reconfigure their collections keeping in view the changing preferences of information users and develop information tools which help in the earliest and easy access to information.

\section{Digital divide and information literacy.}

Many developing countries could not utilize the full potentialities of their people because of illiteracy. In order to utilize the full potentialities of their literate populations, developing countries must initiate massive information literacy programs to empower them and counter their technological impairment. Libraries must gear up to play an important role in imparting information literacy to empower information user communities. Digital divide is even witnessed among professionals who are working in the digital environment. "Despite the rapid spread of the Internet, the gap is growing wider as the technological standards grew even higher. Faster networks, high level machines, more complex software and more capable professionals are required. But in many nations funding is not available to support such development." (7) It is therefore important to identify that a literacy program is designed for whom and what impact it has on the learners. Any information literacy program should be interesting, keep the learner thinking and engaging, make them realize what the have been missing so far and should have rippling effect on the work of participants.

It has been studied that when some individuals are trained and imparted information literacy or exposed to new technological environments, they become star performers. "For 42 years old, A.V. Narayanaswami, a coffee planter in Wayanad, it has been a labour of love to his vacation as a farmer and as a Keralite concerned about the woes of the state's farm sector. His huge data collection currently runs into 1.5 lakhs web pages 
in more than 300 modules. The database covers the state's farm potential, new norms of production, packaging and marketing." (Suchutra 2002, p.3)

If you have very beneficial information sources and helpful plans and convince the people to adopt these, people not only accept these but also help in the diffusion of information "Mohammad Mushtaq a farm expert has helped as many as 265 farmers to start green house technology in Bilaspur, Hamirpur and Mandi regions of Himachal Pradesh. He has set up his own green house n 30,000 square yards in Morni hills in Srimur district where from he expects to earn Rs. 5 lakhs a day in ten years from now. About 10-15 farmers are consulting him everyday". (Tribune 2006)

In India some efforts have been made here and there to bridge the digital divide. The wireless in Local Loop (WLL) technology developed by IIT Chennai has helped in providing the Internet connectivity to 250 community Kiosks that offer these services to over 700,000 people in rural India" (Gaur, 2003, p.108). Keeping in view the size and population of the country, a number of well planned and coordinated initiatives are desired for making the technological infrastructure available and training masses at various levels.

The key to effectively building collections in the digital era is also training of library professional because if they learn how to make most effective use of technology, the latter will automatically get involved in their work culture. They will thus also help to bridge the digital divide by training the information users. Commitment to service and self initiatives to learn new development are also important. If we are really enthusiastic to serve in the digital information revolution, there is no full stop to service opportunities, if we do not want to effectively serve, the service will not stop.

\section{References}

1. Malhan, I.V. and Gulati Anjali, "Knowledge Management Problems of Developing Countries, with Special Reference to India." Information Development 19(3) September 2003, p 211.

2. Two Exabytes of Data Produced Yearly. Computer-Today 16(214) November 2000, p27

3. Conway, Paul,'Preservation in the Digital World." <www.clir.org>

4. Collection Development Policies.<www.dlapr.lib.az.us/cdf/colldev:html>

5. Ficstar Webgrapper <www.ficstar.com/index.html>

6. Milne, Jasmine, "Headline Grabbing devices". Information. World Review 207, November 2004, p23.

7. Libraries and Intellectual Freedom. IFLA/FAIFE World Report Denmark, p9.

8. Suchitra M, "Farmer who grows Data", The Tribune, December 2, 2002, p3.

9. Helping Farmers to change their Fortune, The Tribune, October 12, 2006, p9.

10. Gaur, Ramesh K, "Rethinking the Indian Digital Divide;; Present state of Digitization in Indian Management Libraries." IN Murthy T.A.V ed, Mapping Technology on Libraries and People, Ahmedabad, Inflibnet, 2003, p108 